\title{
ESTRATEGIAS USADAS PARA ALIMENTAR A NIÑOS PREESCOLARES POR MADRES DE UNA ZONA URBANO MARGINAL DE LIMA, PERÚ
}

\author{
Doris Delgado-Pérez ${ }^{1, a}$, Reyna Liria-Dominguez ${ }^{2, b}$
}

\begin{abstract}
RESUMEN
Se exploraron las estrategias que usan las madres de una zona urbano-marginal de Lima, para alimentar a sus hijos de 36 a 66 meses de edad. Estudio cualitativo con método de teoría fundamentada realizado en 30 madres que participaron en 1 de 4 grupos focales y/o 1 de 11 entrevistas a profundidad. Las entrevistas a profundidad y grupos focales, previamente grabadas, fueron transcritas para la identificación de los temas finales. La edad de las madres estuvo entre los 22 y 42 años; $60 \%$ de ellas con educación básica incompleta. Se encontraron como estrategias positivas dar de comer cuando tiene apetito y el manejo de oportunidades para dar de comer. Como estrategias negativas se encontró presionar para que coma y uso de castigo o premio para que termine su comida. Se concluye que las madres utilizaron estrategias positivas y negativas con la finalidad de que sus hijos coman toda su comida servida.
\end{abstract}

Palabras clave: Estrategias; alimentación; Preescolar; Conducta alimentaria; Investigación cualitativa (Fuente DeCS BIREME).

\section{STRATEGIES USED BY MOTHERS TO FEED PRESCHOOL-AGED CHILDREN IN A MARGINAL URBAN AREA OF LIMA, PERU}

\begin{abstract}
This study explored the strategies for feeding preschool-aged children used by mothers in a marginal urban area of Lima. This qualitative, grounded theory method-based study included 30 mothers who participated in 1 of 4 focus group sessions and/or 1 of 11 in-depth interviews. Previously recorded in-depth interviews and focus groups were transcribed to identify the final issues. The mothers' ages ranged from 22 to 42 years, and $60 \%$ had not completed a basic education. The children's ages ranged from 36 to 66 months. The following positive strategies were identified: a) feeding when hungry and b) management of feeding opportunities. The following negative strategies were also identified: a) forcing a child to eat and b) use of punishment or reward to encourage complete food consumption. In conclusion, the mothers in our study cohort employed both positive and negative strategies to encourage food consumption in their children.
\end{abstract}

Key words: Feeding strategies; Preschool; Feeding behavior; Qualitative research (source: MeSH NLM).

\section{INTRODUCCIÓN}

El sobrepeso y la obesidad son un problema mundial, incluyendo al Perú ${ }^{(1,2)}$. Está descrito que la conducta y los hábitos alimentarios se empiezan a establecer en edades tempranas. Las prácticas de alimentación y las formas de dar de comer son importantes en el comportamiento alimentario futuro y control de peso ${ }^{(3-5)}$.
Durante los primeros 5 años de vida, el niño no es autónomo y son los padres los responsables de alimentarlo y decidir qué, cómo y cuándo deben comer. Las prácticas de alimentación se asocian a la costumbre y propiedades percibidas de los alimentos (6,7). La obesidad se ha asociado al comportamiento de los padres sobre el consumo de alimentos en los niños. La permisividad ante el consumo de alimentos no saludables, control y

\footnotetext{
* Esta investigación forma parte del tema de la tesis para optar al grado de doctor en Ciencias de la Salud del autor principal.

1 Centro de Investigación de Bioquímica y Nutrición Alberto Guzmán Barrón, Facultad de Medicina, Universidad Nacional Mayor de San Marcos. Lima, Perú.

2 Instituto de Investigación Nutricional. Lima, Perú.

Nutricionista, magíster en Nutrición; ${ }^{\mathrm{b}}$ nutricionista, doctor en Ciencias en Nutrición Poblacional

Recibido: 14/08/2015 Aprobado: 01/06/2016
}

Citar como: Delgado-Pérez D, Liria - Dominguez R. Estrategias usadas para alimentar a niños preescolares por madres de una zona urbano marginal de Lima, Perú. Rev Peru Med Exp Salud Publica. 2016;33(3):507-12. doi: 10.17843/rpmesp.2016.333.2295 
uso de la presión al momento de comer, puede romper la autonomía del niño a comer cuando tiene hambre ${ }^{(8,9)}$.

En Perú no hay estudios publicados sobre el comportamiento de los padres en la alimentación de sus hijos en edad preescolar; ya sean comportamientos positivos o negativos, especialmente los relacionados con la actitud de las madres frente a la autonomía del niño que puedan influir en los hábitos actuales y futuros ${ }^{(6,7,10)}$.

El objetivo del estudio fue explorar las estrategias que usan las madres de una zona urbano-marginal de Lima, para dar de comer a sus hijos en edad preescolar.

\section{MATERIALES Y MÉTODOS}

Se realizó un estudio cualitativo utilizando el método de teoría fundamentada (11); se exploraron las estrategias de alimentación que las madres utilizan en niños preescolares (36-66 meses) sanos, del Programa No Escolarizado de Educación Inicial (PRONOEI) ubicado en el asentamiento humano Virgen de la Candelaria de Villa María del Triunfo, Lima, Perú. El estudio fue realizado entre octubre y noviembre de 2014 .

Se aplicaron dos técnicas cualitativas: grupos focales (GF) y las entrevistas a profundidad (EP) para triangular los datos obtenidos. Participaron 19 madres en 4 GF y 11 en EP (equiparados ambos por sexo). La muestra final (30 madres) fue alcanzada por saturación teórica.

Las guías de preguntas de GF y EP fueron evaluadas por expertos en el tema y adaptadas luego de un estudio piloto. Los dominios fueron: i) Descripción de alimentos utilizados en las comidas principales; ii) Estrategias para dar de comer en la casa: reacciones positivas, preferencias de alimentos, y iii) Estrategias negativas para dar de comer.

Los GF fueron conducidos por personal previamente entrenado (moderador y observador para tomar notas) en un ambiente abierto con las madres sentadas en círculo. Se hicieron preguntas orientadoras y se motivó la libre participación de las madres. Los GF y EP se realizaron a las madres después de dejar a sus niños en el PRONOEI. Tanto los GF y EP fueron grabados y luego transcritos para su análisis. La duración aproximada de ambas técnicas fue de $60 \mathrm{~min}$. Al final de cada sesión (GF y EP) las madres completaron un breve cuestionario sociodemográfico.

El tratamiento de datos se enfocó en el análisis de contenidos verbales transcritos de los GF y EP, en las expresiones y el análisis de texto usando el método inductivo. La codificación tuvo tres fases: (1) abierta, para determinar los códigos de temas; (2) axial, para determinar subtemas, y (3) selectiva, para determinar temas mediante triangulación de GF y EP. Los temas seleccionados fueron identificados por consenso entre las autoras, con el fin de mostrar los más relevantes de acuerdo a la información obtenida. Todo esto fue procesado en el software Atlas.ti (versión 7.5.4, 2014; GmBh Berlín).

El estudio fue aprobado por el Comité de Ética de la Universidad Nacional Mayor de San Marcos, Lima, Perú. Las madres firmaron un consentimiento informado para participar voluntariamente en el estudio y permitir grabar la EP y GF. Se resguardó la confidencialidad de la información.

\section{RESULTADOS}

El $60 \%$ de madres (18 de 30) tenían educación básica incompleta; 77\% (23) nacieron fuera de la capital, Lima, y el $70 \%$ provinieron de la sierra. Todas vivían con su pareja. Los gastos diarios en alimentación fueron de S/ 17,50 soles (aproximadamente USD 5,80) (Tabla 1).

Emergieron dos temas y, en cada uno, dos subtemas de las estrategias usadas por las madres para alimentar a sus niños, no se encontró diferencias por sexo: $1 .^{\circ}$ Estrategias positivas, en las que se identificaron dos subtemas: (i) Estrategias relacionadas con el apetito y tiempo de consumo y (ii) Manejo de oportunidades que utiliza a su favor la madre. Entre las estrategias negativas, con dos subtemas: (i) Presión que ejercen las madres para que el niño coma, y (ii) Uso de premios/ castigos para lograr que el niño consuma la comida (Figura 1).

Tabla 1. Características de las madres y de sus hijos participantes en los grupos focales o entrevistas a profundidad de una zona urbano-marginal de Lima $(n=30)$

\begin{tabular}{|c|c|c|}
\hline \multirow{2}{*}{$\begin{array}{ll} & \text { Características } \\
\text { Edad }^{*} & \end{array}$} & \multicolumn{2}{|c|}{ n (\%) } \\
\hline & 30,3 & $(22-42)$ \\
\hline Tiempo de residencia en Lima (años) ${ }^{\star}$ & 12,8 & $(0,4-41)$ \\
\hline \multicolumn{3}{|l|}{ Educación, n (\%) } \\
\hline Primaria & 10 & $(33,3)$ \\
\hline Secundaria & 18 & $(60,0)$ \\
\hline Técnico & 2 & $(6,7)$ \\
\hline Número de hijos* & 2,2 & $(1-3)$ \\
\hline $\begin{array}{l}\text { Gastos diarios - alimentación (S/), } \\
\text { promedio (rango) }\end{array}$ & 17,5 & $(10-25)$ \\
\hline Peso materno $(\mathrm{kg})^{*}$ & 57,8 & $(45-78)$ \\
\hline \multicolumn{3}{|l|}{ Niños } \\
\hline Edad en meses* & 56,0 & $(44-66)$ \\
\hline \multicolumn{3}{|l|}{ Sexo, n (\%) } \\
\hline Varones & 15 & $(50,0)$ \\
\hline Mujeres & 15 & $(50,0)$ \\
\hline
\end{tabular}




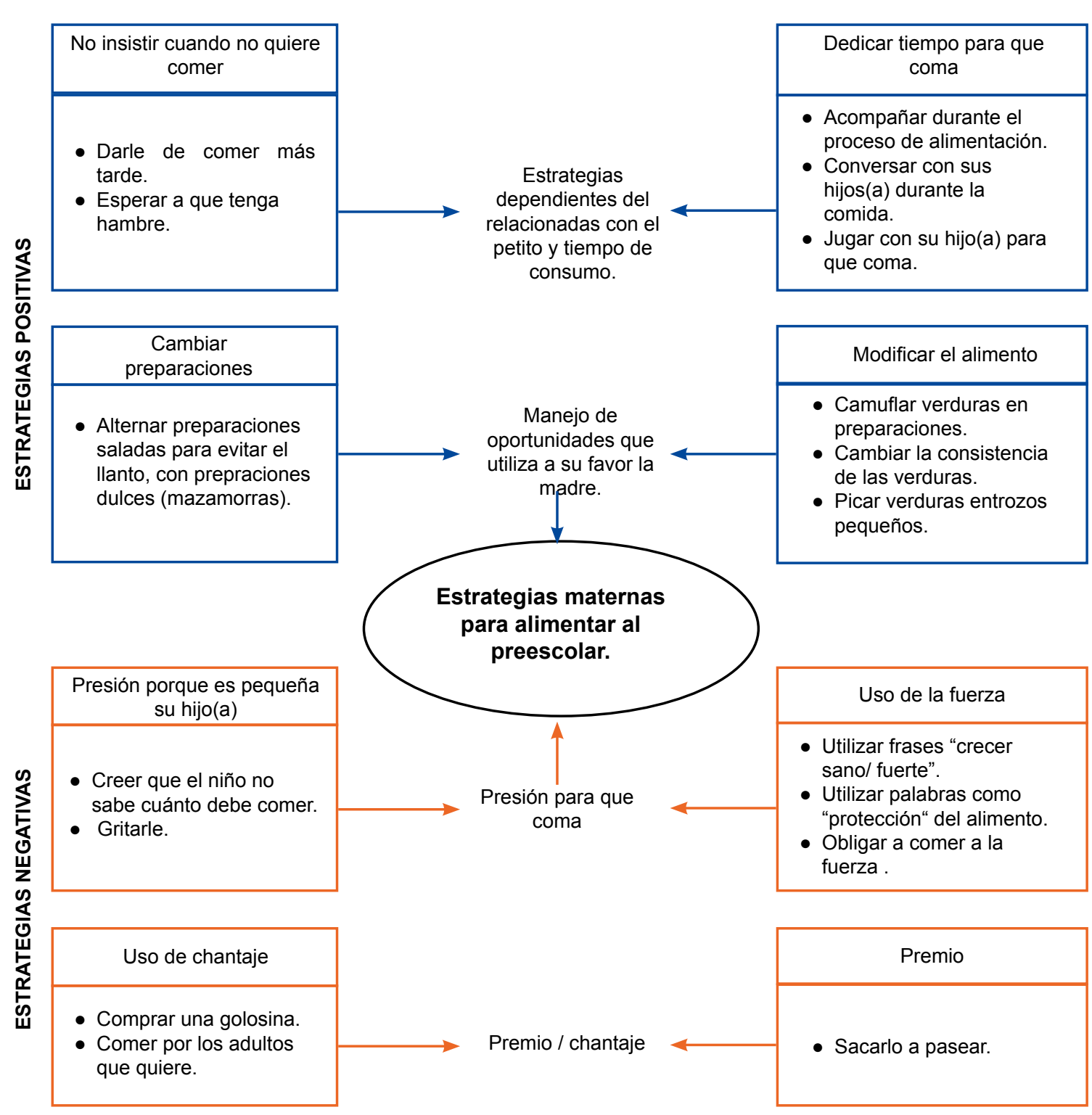

Figura 1. Estrategias positivas y negativas maternas para dar de comer a sus hijos preescolares de una zona urbanomarginal, Lima $(n=30)$

Tema 1. Estrategias positivas de dar de comer

Entre las estrategias positivas para incentivar y promover el consumo de alimentos en el niño.

Subtema 1.1. Estrategias relacionadas con el apetito y tiempo de consumo

Las madres consideraron que no se debe insistir cuando el niño no desea comer, o esperar a que tenga hambre para darle de comer. Ellas mencionaron que su hijo no tiene apetito cuando llegan del colegio, por lo que prefieren servir la comida más tarde cuando tiene hambre, para que coman mejor (Tabla 2: 1.1.1 y 1.1.2).
Asimismo, las madres mencionaron dedicar tiempo a sus niños usando diferentes estrategias positivas: i) brindarle compañía durante el tiempo de comida;

ii) Conversar motivándolo a comer, y iii) interactuar mediante el juego (Tabla 2: 1.1.3-1.1.5).

Subtema 1.2. Manejo de oportunidades que la madre utiliza a su favor

Las madres manifestaron algunas estrategias usadas para que el niño consuma una dieta saludable: i) Cambiar la forma de preparar alimentos que no les gustan a los niños, pero que ellas consideran saludables. Una de las madres reveló que utilizaba la quinua en preparaciones 
Tabla 2. Ejemplos de citas que apoyan al primer tema: "Estrategias positivas para dar de comer" y segundo tema "Estrategias negativas para dar de comer" que utilizan las madres con hijo(a) preescolar de una zona urbano-marginal, Lima $(n=30)$

\begin{tabular}{|c|c|}
\hline $\mathbf{N}^{\mathbf{0}}$ & Temas/Citas \\
\hline 1 & Tema 1: Estrategias positivas para dar de comer \\
\hline Subtema 1.1 & Estrategias relacionadas con el apetito y tiempo de consumo \\
\hline 1.1 .1 & $\begin{array}{l}\text { l... cuando llega del colegio me dedico a ella para que coma, porque a veces no tiene apetito, se va el hambre. } \\
\ldots \text { yo me dedico, sentada para que coma/. (EP). }\end{array}$ \\
\hline 1.1 .2 & $\begin{array}{l}\text { I... le hago comer de nuevo, después de tres horas o dos horas, le hago comer otra vuelta, le hago comer una } \\
\text { fruta. Comerá la cuarta parte o quinta parte/. (GF). }\end{array}$ \\
\hline 1.1 .3 & I... cuando llega del colegio me dedico a ella para que comal. (GF). \\
\hline 1.1 .4 & I... pero si tu pones a comer a su lado le dices que esta rico, si come/. (GF). \\
\hline 1.1 .5 & I...jugando le doy, como sea le tengo que dar de comer/. (EP). \\
\hline Subtema 1.2 & Manejo de oportunidades que utiliza a sus favor la madre \\
\hline 1.2 .1 & $\begin{array}{l}\text { l... no, le dijo este, (refiriéndose a la quinua) si no quiere en sopa le hago en mazamorra, o en desayuno, o en } \\
\text { sopa, sí o sí le hago comer/. (GF). }\end{array}$ \\
\hline 1.2 .2 & I... a la espinaca lo hago en torreja eso le gusta o en guiso/. (EP) \\
\hline 1.2 .3 & $\begin{array}{l}\text { I... espinaca, picadito, para que come, a veces le doy grande no quiere/. } \\
\text { /... al brócoli lo hago como puré/. (EP). }\end{array}$ \\
\hline 2 & Tema 2: Estrategias negativas para dar de comer \\
\hline Subtema 2.1 & Presión para que el niño(a) coma \\
\hline 2.1 .1 & $\begin{array}{l}\text { l... no, no va hacer lo que le conviene, no lo que ella quiere. Yo le agrego, la comida que creo conveniente/. } \\
\text { (GF). }\end{array}$ \\
\hline 2.1 .2 & $\begin{array}{l}\text { I... yo o sea, cuando no quiere comer, por ejemplo, si no quiero comer la quinua, normalmente yo le obligo } \\
\text { porque es pequeña/. (EP). }\end{array}$ \\
\hline 2.1 .3 & I... cuando no quiere comer le gritamos, hace su pataleta, llorando come/. (GF). \\
\hline 2.1 .4 & $\begin{array}{l}\text { I... a la fuerza le hago comer, yo le digo tienes que comer, quieres crecer sana y fuerte, sí, ahí recién come, } \\
\text { tengo que estar amenazándola, porque no le gusta, el olor es desagradable/. (EP). }\end{array}$ \\
\hline 2.1 .5 & $\begin{array}{l}\text { l... pero si no quiere la quinua yo le hago comer a la fuerza tres o cuatro cucharadas. Pero yo le digo que te a } \\
\text { proteger el alimento, la quinua es para que crezca fuerte/. (EP). }\end{array}$ \\
\hline 2.1.6 & I... si a la fuerza tiene que comer/. (EP). \\
\hline Subtema 2.2 & Uso de premio/chantaje \\
\hline 2.2 .1 & $\begin{array}{l}\text { I... cuando no quiere comer, yo siempre le digo me quieres, por mí, por su papá, por su abuelita. Menos de } \\
\text { medio plato no me termina, siempre tiene que ser un platito/. (GF). }\end{array}$ \\
\hline 2.2 .2 & $\begin{array}{l}\text { l... al ratito déjalo, después me dice mami quiero comprar un dulce, ella me pide comprar, le dijo que primero es } \\
\text { la comida, después le digo que es la golosina/. (EP). }\end{array}$ \\
\hline 2.2 .3 & I....como sea le tengo que dar de comer, le digo ya no te saco a la calle, así le doy de comer/. (EP). \\
\hline 2.2 .4 & $\begin{array}{l}\text { l... me siento a veces mal, a veces bien, porque es un alimento que no come, pienso que no come, porque va } \\
\text { ser así toda la vida/. (EP). }\end{array}$ \\
\hline
\end{tabular}

$E P=$ Entrevista a profundidad.

$\mathrm{GF}=$ Grupo focal

saladas o dulces, especialmente en las comidas principales (Tabla 2: 1.2.1). ii) Modificar los alimentos, por ejemplo camuflan verduras dentro de preparaciones preferidas, o las pican muy pequeño. Aunque no todas las estrategias a este nivel fueron saludables, algunas freían los alimentos para que los niños los consuman, y iii) Modificar la consistencia del alimento, por ejemplo haciéndola puré (Tabla 2: 1.2.2 y 1.2.3).

Tema 2. Estrategias negativas para dar de comer

Una de las mayores preocupaciones de las madres es que su niño crezca sano y fuerte, por eso, algunas veces manifestaron que utilizaban estrategias que podríamos considerarlas negativas: 1) presionar para que coman, y 2) usar premio/chantaje.
Subtema 2.1. Presión para que coma

Las madres consideraban que por ser pequeños sus hijos no saben lo que les conviene, por eso es necesario obligarlos a comer. Algunas utilizaban la fuerza para que termine su comida, pudiendo optar por: i) Gritar, el niño llora comiendo lo que no le gusta, y ii) Forzar o presionar a comer todo o al menos una parte (Tabla 2: 2.1.1 - 2.1.6).

\section{Subtema 2.2. Uso de premio/chantaje}

Algunas madres manifestaron que usaron el premio o chantaje para que el niño termine su comida, por ejemplo: i) Decirles que si comen quiere a su familiar, especialmente a sus padres; ii) Si termina le compra una golosina, y iii) Si termina lo saca a pasear (Tabla 2: 2.2 .1 - 2.2.3). 
Sin embargo, el uso de estrategias negativas las hace sentirse mal por la actitud que tomaron, pero, a la vez, se sienten mal porque a pesar del esfuerzo económico sus hijos no quieren comer (Tabla 2: 2.2.4).

\section{DISCUSIÓN}

El presente estudio cualitativo encontró que las madres con niños en edad preescolar usaban: $1 .^{\circ}$ Estrategias positivas como: (i) Estrategias relacionadas con el apetito y tiempo de consumo, y (ii) Manejo de oportunidades; y 2. ${ }^{\circ}$ Estrategias negativas como: (i) Presionar al niño para que coma, y (ii) Uso de premio/chantaje.

En los primeros años de vida el ambiente familiar, el comportamiento de la madre y los hábitos de consumo influyen en las preferencias alimentarias de sus hijos ${ }^{(12)}$. Estos son de vital importancia porque se han asociado con sobrepeso y obesidad en la adolescencia y edad adulta.

\section{Reacciones positivas para dar de comer}

Las madres mencionaron dedicar tiempo a sus hijos, acompañándolos, conversándoles o interactuando, para que crezcan sanos. Un estudio con madres mexicanas migrantes en Estados Unidos, menciona que ellas acompañaban y alimentaban a su hijos en edad preescolar, haciendo placentero el momento de comer ${ }^{(13)}$.

Muy pocas madres permitieron autonomía en el niño. Manifestaron no insistir cuando el niño no quiere comer e identifican cuando tiene o no hambre y, de acuerdo a esto, le sirven. Además, si no quiere comer, no insisten y le ofrecen más tarde. Pero, la mayoría consideraba importante que el niño termine su plato de comida por lo que ejercieron presión. Sherry et al. reportaron que la madre insiste hasta que el niño termine la cantidad servida, a pesar de que éste mencione estar "lleno" (14). Croker et al. mencionan que las madres detectan las señales del hijo cuando desea comer más y le sirven más ${ }^{(15)}$. Se ha descrito que las estrategias adoptadas por las madres dependen de cómo conciben a sus hijos, ya sea como buenos o malos consumidores. Las madres que los consideran buenos consumidores (porque consumen comida saludable), no tienen problemas. Pero las que los consideran malos consumidores, prefieren que coma, aunque solo sea lo que le gusta, con tal que coma ${ }^{(16)}$. Otros autores mencionan que los niños pueden mostrar aversión a ciertas texturas y consistencia de alimentos, probablemente por la sensación que les da al tenerlos en la boca ${ }^{(17)}$.

Las madres evocan alimentos de la región donde nacieron, porque los consideraban saludables e importantes para sus hijos (quinua, maíz, trigo). Algunos niños expresaron su disgusto a estos y otros alimentos llorando. En estos casos las madres decidieron prepararlos de diferente manera: enmascarar $y$ camuflándolos con otros alimentos, o modificando su consistencia. Diversos autores indican que madres de bajo nivel socioeconómico consideran la alimentación con una alta carga emocional y buscan incorporarlos en preparaciones que los niños consuman (13); esto sucede en especial con madres latinoamericanas ${ }^{(10,18)}$.

\section{Reacciones negativas para dar de comer}

Las madres se sintieron frustradas, porque a pesar de usar diferentes estrategias algunos niños no terminan su ración servida. Ante esta situación la madre pierde la paciencia y reacciona negativamente. Algunas usaban la fuerza o levantaban la voz para presionar y lograr que terminen sus alimentos regionales, verduras $y$ leguminosas. Muchos autores mencionan las verduras como uno de los grupos de alimentos con mayor rechazo ${ }^{(15,18)}$. Además, resaltan que la introducción de nuevos alimentos y la presión de los padres, crea neofobia alimentaria en los niños, es decir aversión a estos alimentos ${ }^{(19)}$.

El chantaje fue otra estrategia negativa usada por las madres. Ofrecen un alimento no saludable (golosinas) para después de terminar su comida. Diversos estudios mencionan que el uso del chantaje por un lado puede provocar que el niño consuma más de lo necesario y, por el otro, puede distorsionar la sensación de sentirse satisfecho ${ }^{(6,10)}$.

Las estrategias negativas usadas por las madres les provocaban un conflicto consigo mismas, porque consideraron que no deberían comportarse así, pero lo hacen para lograr que sus hijos crezcan sanos y fuertes. Algunos estudios explican que algunas madres no repiten lo que vivieron, por eso tratan de hacer que el momento de comer sea lo más placentero posible ${ }^{(13,20)}$.

Nuestro estudio tiene limitaciones, no se recolectó información sobre el trato que recibieron las madres cuando eran pequeñas en el proceso de alimentación que, probablemente, pueda afectar en el uso de las estrategias. Así mismo, los resultados representan a un grupo de madres de un centro de educación inicial de una zona urbano-marginal. Pero, ante la poca información, el presente estudio, al tener una muestra alcanzada por saturación teórica, permitió conocer a profundidad los temas emergentes aportando valiosa información.

En conclusión, los resultados de este estudio muestran que las madres utilizaron tanto estrategias positivas en forma responsiva, como negativas, en forma de presión, chantaje para que sus hijos consuman la cantidad que ellas consideran adecuada. Estos hallazgos 
pueden ayudar a proponer y evaluar intervenciones de alimentación para el preescolar que, por un lado logren un crecimiento adecuado, pero por el otro prevengan problemas nutricionales en la edad adulta. Hace falta investigar en diferentes poblaciones del Perú.

Contribuciones de autoría: DDP ha participado en el concepto y diseño del estudio, recolección de datos y obtención de resultados, análisis e interpretación delos datos, redacción del artículo, revisión crítica del artículo, aprobación de su versión final. RLD ha participado en el concepto y diseño del estudio, análisis e interpretación de los datos, redacción del artículo, revisión crítica del artículo, aprobación de su versión final.

Fuentes de financiamiento: autofinanciado.

Conflictos de interés: los autores declaran no tener conflictos de interés en la publicación de este trabajo.

\section{REFERENCIAS BIBLIOGRÁFICAS}

1. de Onis M, Blössner M, Borghi E. Global prevalence and trends of overweight and obesity among preschool children. Am J Clin Nutr. 2010;92(5):1257-64. doi: 10.3945/ajen.2010.29786.

2. Instituto Nacional de Estadística e Informática. Encuesta Demográfica y de Salud Familiar 2014. Lima: INEI; 2015.

3. Carnell S, Cooke L, Cheng $\mathrm{R}$, Robbins A, Wardle J. Parental feeding behaviours and motivations. A qualitative study in mothers of UK preschoolers. Appetite. 2011;57(3):65573. doi: 10.1016/j.appet.2011.08.009.

4. Haycraft EL, Blissett JM. Maternal and paternal controlling feeding practices: reliability and relationships with BMI. Obesity (Silver Spring). 2008;16(7):1552-58. doi: 10.1038/ oby.2008.238.

5. Domínguez-Vásquez $\mathrm{P}$, Olivares $\mathrm{S}$, Santos JL. Influencia familiar sobre la conducta alimentaria y su relación con la obesidad infantil. ALAN. 2008;58(3):249-55.

6. Lindsay AC, Sussner KM, Greaney ML, Peterson KE. Latina mothers' beliefs and practices related to weight status, feeding, and the development of child overweight. Public Health Nurs. 2011;28(2):107-18. doi: 10.1111/j.1525-1446.2010.00906.x.

7. Mazarello Paes V, Ong KK, Lakshman R. Factors influencing obesogenic dietary intake in young children (0-6 years): systematic review of qualitative evidence. BMJ Open. 2015;5(9):e007396. doi: 10.1136/ bmjopen-2014-007396.

8. Fulkerson JA, Kubik MY, Rydell S, Boutelle KN, Garwick A, Story M, et al. Focus groups with working parents of school-age children: What's needed to improve family meals? J Nutr Educ Behav. 2011;43(3):189-93. doi: 10.1016/j.jneb.2010.03.006.
9. Rodriguez-Oliveros G, Haines J, Ortega-Altamirano D, Power E, Taveras EM, González-Unzaga MA, et al. Obesity determinants in Mexican preschool children: parental perceptions and practices related to feeding and physical activity. Arch Med Res. 2011;42(6):532-9. doi: 10.1016/j. arcmed.2011.10.006.

10. Sanches MS, Andrade RM. Percepciones y prácticas de madres de pre-escolares brasileños acerca de la alimentación de los hijos. Enferm glob [Internet]. 2011 enero [citado el 19 de julio de 2016];10(1): [11 pág.]. Disponible en : http://revistas.um.es/ eglobal/article/view/115871

11. Maxwell JA. Qualitative Research Design: An interactive approach. $3^{\mathrm{a}} \mathrm{ed}$. Washington, D.C.: SAGE; 2012.

12. Dattilo AM, Birch L, Krebs NF, Lake A, Taveras EM, Saavedra JM. Need for early iterventions in the prevention of pediatric overweight: a review and upcoming directions. J Obes. 2012;2012:123023. doi: $10.1155 / 2012 / 123023$.

13. Kalinowski A, Krause K, Berdejo C, Harrell K, Rosenblum K, Lumeng JC. Beliefs about the role of parenting in feeding and childhood obesity among mothers of lower socioeconomic status. J Nutr Educ Behav. 2012;44(5):432-7. doi: 10.1016/j.jneb.2010.09.017.

14. Sherry B, McDivitt J, Birch LL, Cook FH, Sanders S, Prish JL, et al. Attitudes, practices, and concerns about child feeding and child weight status among socioeconomically diverse white, Hispanic, and AfricanAmerican mothers. J Hum Nutr Diet. 2004;104(2):215-21.

15. Croker H, Sweetman C, Cooke L. Mothers' views on portion sizes for children. J Hum Nutr Diet. 2009;22(5):437-43. doi: 10.1111/j.1365-277X.2009.00969.x.
16. Moore SN, Tapper K, Murphy S Feeding goals sought by mothers of 3-5-year-old children. $\mathrm{Br} \mathrm{J}$ Health Psychol. 2010;15(1):185-96. doi: $10.1348 / 135910709$ X447668.

17. Nederkoorn C, Jansen A, Havermans RC. Feel your food. The influence of tactile sensitivity on picky eating in children. Appetite. 2015;84:7-10. doi: 10.1016/j.appet.2014.09.014.

18. Martinez SM, Rhee $\mathrm{K}$, Blanco E, Boutelle K. Maternal attitudes and behaviors regarding feeding practices in elementary school-aged Latino children: a pilot qualitative study on the impact of the cultural role of mothers in the US-Mexican border region of San Diego, California. J Acad Nutr Diet. 2014;114(2):230-7. doi: 10.1016/j.jand.2013.09.028.

19. Kaar JL, Shapiro AL, Fell DM, Johnson SL. Parental Feeding Practices, Food Neophobia, and Child Food Preferences: What combination of factors results in children eating a variety of foods? Food Qual Prefer. 2016;50:57-64. doi:10.1016/j. foodqual.2016.01.006.

20. Malhotra K, Herman AN, Wright G, Bruton Y, Fisher JO, Whitaker RC. Perceived benefits and challenges for low-income mothers of having family meals with preschool-aged children: childhood memories matter. J Acd Nutr Diet. 2013;113(11):1484-93. doi: 10.1016/j.jand.2013.07.028.

Correspondencia: Doris Hilda Delgado Pérez Dirección: Av. Graucuadra 11 - La Victoria, Lima, Perú.

Teléfono: (511) 6197000 - Anexo 4698

Correo electrónico:ddelgadop@unmsm.edu.pe 